\title{
Optical Properties and Quasiparticle Band Gaps of Transition-Metal Atoms Encapsulated by Silicon Cages
}

M. I. A. Oliveira, R. Rivelino, F. de Brito Mota and Gueorgui Kostov Gueorguiev

\section{Linköping University Post Print}

\section{Tweet}

N.B.: When citing this work, cite the original article.

Original Publication:

M. I. A. Oliveira, R. Rivelino, F. de Brito Mota and Gueorgui Kostov Gueorguiev, Optical Properties and Quasiparticle Band Gaps of Transition-Metal Atoms Encapsulated by Silicon Cages, 2014, The Journal of Physical Chemistry C, (118), 10, 5501-5509.

http://dx.doi.org/10.1021/jp409967a

Copyright: American Chemical Society http://pubs.acs.org/

Postprint available at: Linköping University Electronic Press http://urn.kb.se/resolve?urn=urn:nbn:se:liu:diva-106020 


\title{
Optical Properties and Quasiparticle Band Gaps of Transition-Metal Atoms Encapsulated by Silicon Cages
}

\author{
M. I. A. Oliveira ${ }^{\dagger, \star}$, R. Rivelino*,†, F. de Brito Mota ${ }^{\dagger}$, and G. K. Gueorguiev $*, \ddagger$ \\ †Instituto de Física, Universidade Federal da Bahia, 40210-340 Salvador, Bahia, Brazil \\ Department of Physics, Chemistry and Biology (IFM), Linköping University, S-581 83 \\ Linköping, Sweden
}

\begin{abstract}
Semiconductors assembled upon nano-templates consisting of metalencapsulating Si cage clusters $\left(\mathrm{M} @ \mathrm{Si}_{n}\right)$ have been proposed as prospective materials for nano-devices. To make an accurate and systematic prediction of the optical properties of such $\mathrm{M} @ \mathrm{Si}_{n}$ clusters, which represent a new type of metal-silicon hybrid material for components in nanoelectronics, we have performed first-principles calculations of the electronic properties and quasiparticle band gaps for a variety of $\mathrm{M} @ \mathrm{Si}_{12}(\mathrm{M}=\mathrm{Ti}, \mathrm{Cr}, \mathrm{Zr}$, $\mathrm{Mo}, \mathrm{Ru}, \mathrm{Pd}, \mathrm{Hf}$, and $\mathrm{Os})$ and $\mathrm{M} @ \mathrm{Si}_{16}(\mathrm{M}=\mathrm{Ti}, \mathrm{Zr}$, and $\mathrm{Hf})$ clusters. At first stage, the electronic structure calculations have been performed within plane-wave density functional theory in order to predict equilibrium geometries, polarizabilities, and optical absorption spectra of these endohedral cage-like clusters. The quasiparticle calculations were performed within the $G W$ approximation, which predict that all these systems are semiconductors exhibiting large band gaps. The present results have demonstrated that the independentparticle absorption spectra of $\mathrm{M} @ \mathrm{Si}_{n}$, calculated within the local density or generalized gradient approximations to density-functional theory, are dramatically influenced by manybody effects. On average, the quasiparticle band gaps were significantly increased, in comparison with the independent-particle gaps, giving values in the $2.45-5.64 \mathrm{eV}$ range. Consequently, the inclusion of many-body effects in the electron-electron interaction, and going beyond the mean-field approximation of independent particles, might be essential to realistically describe the optical spectra of isolated $\mathrm{M} @ \mathrm{Si}_{n}$ clusters, as well as their clusterassembled materials.
\end{abstract}




\section{INTRODUCTION}

Electronic and structural properties of transition metal atoms encapsulated in cagelike silicon clusters have been extensively studied, due to the possibility of these systems being employed as building blocks for cluster-assembled semiconducting materials. ${ }^{1-7}$ Early studies on the formation of these cluster-metal atom compounds in a supersonic molecular beam has been reported by Beck. ${ }^{8}$ From the technological viewpoint, as in the case of most

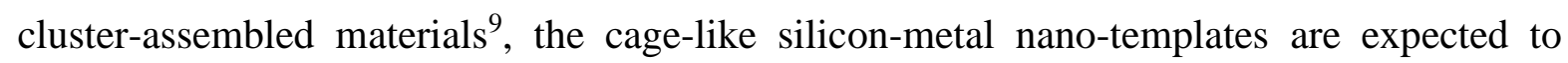
exhibit distinct optical properties from those of pure silicon clusters ${ }^{10}$ or metals. Currently, silicon is the chemical element which is most widely used in microelectronics. In turn, the incorporation of metal atoms into endohedral $\mathrm{Si}$ clusters stabilizes them and makes the corresponding condensed phases semiconductors feasible and efficient for several applications, such as light-emitting diodes, photovoltaic solar cells, thin-film transistors and other photoelectronic devices ${ }^{11,12}$. Actually, these systems have been considered ideal candidates for the next generation of silicon-based nanoelectronics. The stability exhibited by endohedral Si cages, when their internal dangling bonds are recombined with $d$ orbitals by the incorporation of a transition-metal atom (M) at its geometric center, as first shown by Hiura et $a l .{ }^{13}$, is an important motivation for further studies dedicated to these systems.

Systematic studies about the dependency on size (number of silicon atoms, $n$ ) as well as on the transition metal element indicate that clusters with $n=7,10,12$ exhibit high cohesive energies, independently of the metal species involved ${ }^{2,4}$. In contrast to the emblematic clusters with twelve silicon atoms, which exhibit the characteristic conformation of a hexagonal prism (a cluster that, in its undistorted shape, belongs to the $D_{6}$ point group symmetry) for all $\mathrm{M}$ species, most of the other energetically favorable $\mathrm{M}_{0} \mathrm{Si}_{n}(n<12)$ clusters do not exhibit any special symmetry. ${ }^{2,4,14}$ However, in their "as synthesized" form, many of the M@Si 12 clusters are slightly distorted from the ideal $D_{6 \mathrm{~h}}$ symmetry. ${ }^{13}$ More interestingly, the $\mathrm{M} @ \mathrm{Si}_{12}$ clusters synthesized in the gas phase have been successfully deposited on Si-substrates. ${ }^{15}$ Also, as a stable Si-based alternative of the carbon nanotubes, nanowires with metallic properties built up from $\mathrm{M} @ \mathrm{Si}_{12}$ clusters have been predicted. ${ }^{16}$

Another class of interesting $\mathrm{M} @ \mathrm{Si}_{n}$ systems, which has received special attention in the recent years, is that of the $\mathrm{M} @ \mathrm{Si}_{16}$ clusters $(\mathrm{M}=\mathrm{Ti}, \mathrm{Zr}$ and $\mathrm{Hf}) .{ }^{17-18}$ Similarly to the case of $\mathrm{M} @ \mathrm{Si}_{12}$, these systems have been considered as promising units for assembling optoelectronic devices. ${ }^{19-20}$ The strong covalent bond between the metal-center (e.g., Ti, $\mathrm{Zr}$ and $\mathrm{Hf}$ ) and the $\mathrm{Si}_{16}$ cage leads to larger gaps, exhibiting absorption in the visible region. ${ }^{21,22}$ As widely accepted in the literature, these features of $\mathrm{M} @ \mathrm{Si}_{16}$ emphasize their generally 
higher stability when compared to $\mathrm{M} @ \mathrm{Si}_{12},{ }^{5,7,17-22}$ making $\mathrm{M} @ \mathrm{Si}_{16}$ possibly more eligible than $\mathrm{M} @ \mathrm{Si}_{12}$ for viable applications. Thus, the structural stability of these clusters is a significant advantage for possible applications of $\mathrm{M} @ \mathrm{Si}_{16}$ in optical devices. Furthermore, the oligomerization of two fullerene-like $\mathrm{Zr} @ \mathrm{Si}_{16}$ and two Frank-Kasper-like Ti@ $\mathrm{Si}_{16}$ clusters has demonstrated that the dimers keep the cage-structures of the clusters intact, which confirms the possibility of self-organized assemblies of these superatoms. ${ }^{23}$

In general, one may expect that the silicon cage clusters doped with transition metal atoms also exhibit some similarities with endohedral fullerenes encapsulating metal atoms. Particularly, studies on the polarizability of these clusters may reveal a closer relationship with their photophysical properties. For instance, in the context of endohedral fullerenes, Sabirov and Bulgakov ${ }^{24}$ have investigated the polarizability magnification attributed to the encapsulation effect. Also, interesting electronic properties of small alkali-based metallofullerenes, e.g., $\mathbf{M} @ \mathrm{C}_{20}$, have been studied through ab initio methods. ${ }^{25,26}$ Recently, theoretical predictions of metallofullerenes containing transition metal atoms have indicated that their electronic and optical properties can be properly tuned. ${ }^{27,28}$ All these features also deserve to be carefully evaluated in the context of the silicon cage clusters containing transition metal atoms.

Although the stable and synthesizable metal-encapsulating Si clusters have been considered and discussed as potential candidates for optical nanodevices, their excited state properties have been scarcely addressed. ${ }^{29}$ In contrast, the optical properties of silicon quantum dots, ${ }^{30}$ silicon nanocrystals ${ }^{31}$ and small silicon clusters ${ }^{32,33}$ have been extensively described by using different levels of theory. These theoretical methods range from the independent-particle model up to more accurate approaches employed in the optical spectrum calculations, such as the $G W$ approximation (GWA). ${ }^{34}$ Hence, in addition to determining the ground-state properties of some important $\mathrm{M} @ \mathrm{Si}_{n}$ clusters, within at a high level of theory, our main purpose here is to understand the role of many-body effects in the optical properties of these potential building blocks for cluster-assembled semiconducting materials.

In the present work, we report accurate results on the polarizabilities, absorption spectra, and quasiparticle band gaps of stable $\mathrm{M} @ \mathrm{Si}_{12}(\mathrm{M}=\mathrm{Ti}, \mathrm{Cr}, \mathrm{Zr}, \mathrm{Mo}, \mathrm{Ru}, \mathrm{Pd}, \mathrm{Hf}$, and Os) and $\mathrm{M} @ \mathrm{Si}_{16}(\mathrm{M}=\mathrm{Ti}, \mathrm{Zr}$, and $\mathrm{Hf}$ ) clusters, obtained from first-principles densityfunctional theory (DFT) calculations. Adopting a more sophisticated theoretical approach to realistically describe the quasiparticle (QP) spectra of different $\mathrm{M} @ \mathrm{Si}_{n}$ systems, we have employed the many-body Green's function perturbation formalism within GWA. Starting from the local density approximation (LDA) ${ }^{35}$ to DFT, the total energies have been 
evaluated by employing the scheme based on the projector augmented wave basis sets ${ }^{36}$ method. Our results are discussed here in comparison with the ground state properties of these systems (also calculated in this work), and compared to recent results for correlated systems obtained from the literature.

\section{METHODOLOGY AND COMPUTATIONAL DETAILS}

2.1. Gradient Optimizations and Ground-State Description. Geometry optimizations and electronic-structure calculations of the $\mathrm{M} @ \mathrm{Si}_{12}(\mathrm{M}=\mathrm{Ti}, \mathrm{Cr}, \mathrm{Zr}, \mathrm{Mo}, \mathrm{Ru}, \mathrm{Pd}, \mathrm{Hf}$, and Os) and $\mathrm{M} @ \mathrm{Si}_{16}(\mathrm{M}=\mathrm{Ti}, \mathrm{Zr}$, and $\mathrm{Hf})$ clusters were carried out by employing plane-wave DFT calculations, as implemented in the VASP code. ${ }^{36-38}$ The starting point geometries of M@Si 12 were considered within the $D_{6 \mathrm{~h}}$ symmetry and those of M@Si 16 were considered within the $C_{3 \mathrm{v}}$ symmetry ${ }^{39}$ (Frank-Kasper type structure). The exchange-correlation potential was approximated by the generalized gradient approximation (GGA), using the PerdewWang (PW91) scheme ${ }^{40}$ with the full-potential projector augmented wave (PAW) method $^{41,42}$ and $380 \mathrm{eV}$ as plane wave cutoff. Hence, all cluster geometries were fully optimized at the PW91/PAW level of theory. For the gradient optimizations, an automatic mesh to sample only the $\Gamma$ point of the Brillouin zone was employed. Cubic boxes with side of $15 \AA$ for $\mathrm{M} @ \mathrm{Si}_{12}$ and $20 \AA$ for $\mathrm{M} @ \mathrm{Si}_{16}$ were employed to avoid interactions with adjacent images. The atomic positions were optimized within $0.01 \mathrm{eV} / \AA$ for the atomic forces and the convergence criterion for the total electronic energy calculations was set to $10^{-5} \mathrm{eV}$. The choice of the VASP code to optimize the M@Si $\mathrm{i}_{n}$ clusters reported in this work was due to the high accuracy of the PAW method (especially when dealing with metal atoms and even when the atomic core wave functions are included ${ }^{42}$ ). Furthermore, to evaluate spin effects on the electronic properties of these systems, spin-polarized calculations have been performed within this same level of theory.

\subsection{Bader Charge Analysis, Finite Field Method and Random Phase Approximation. In} order to characterize the electric properties of the $\mathrm{M} @ \mathrm{Si}_{n}$ clusters, as well as to accurately predict their optical properties, we have calculated charge distributions, dipole moments, and static polarizabilities. This information is of great value for further experimental studies of such systems, as reported by Broyer et $a l .{ }^{43}$ in the context of other metal-cage clusters. The charge analysis was performed within the Bader method ${ }^{44}$ considering a charge density grid in the VASP CHGCAR format. From this analysis, we have obtained the charge 
transfer direction between the metal and silicon cage in each $\mathrm{M} @ \mathrm{Si}_{\mathrm{n}}$ cluster. The dipole moments and static polarizability tensors were calculated within the finite field method ${ }^{45,46}$ in the approximation of small electric fields applied in the three Cartesian directions. Furthermore, the dynamic polarizabilities were calculated within the random-phase approximation (RPA), as implemented in the VASP code, taking into account 300 bands in the frequency dependent polarizability, which was evaluated up to $10 \mathrm{eV}$. In this scheme, the frequency dependent dielectric matrix was calculated from the electronic ground state. The imaginary part was determined by a summation over empty Kohn-Sham states, while the real part of the dielectric tensor was obtained by the usual Kramers-Kronig transformation. ${ }^{37,38}$

\subsection{Quasiparticle Calculations within the Frequency-Dependent GW Approximation. The} quasiparticle $(\mathrm{QP})$ band gaps of the $\mathrm{M} @ \mathrm{Si}_{n}$ cages were calculated starting from DFT calculations within LDA to obtain the Kohn-Sham (KS) eigenvalues and eigenvectors, which provide the independent-particle absorption spectrum. In this case, we have considered the same optimization criteria (with a plane wave kinetic energy cutoff of 340 $\mathrm{eV})$ as described above. The treatment of semicore states in these calculations was considered, which is especially important in the cases of Ti and Ru. For the QP calculations, we have employed the frequency-dependent $G W$ method within the PAW framework, as implemented in the VASP code. ${ }^{37,38,47}$ Using this approach, the vertex corrections were not taken into account and the self-energy $\Sigma$ operator was reduced to a single direct product of one-particle Green's function $G$ and the screened Coulomb interaction $W$. The iterative procedure constructs $\Sigma=i G W$ from the KS eigenvalues and eigenfunctions. To correct the band gap values obtained with LDA, we have started the calculations from a single shot $G_{0} W_{0}$. Then, the set of QP shifts were iterated four times whereas the screened Coulomb potential $W$ was fixed to the initial DFT $W_{0}$, and only the eigenvalues for the one-particle Green's function were updated (usually called $\left.G W_{0}\right) .{ }^{47,48}$ As it is well known, fully selfconsistent calculations deteriorate the QP spectrum and yield overestimated gaps. ${ }^{42}$ During the frequency-dependent GWA calculations, we have employed 300 bands, 80 frequency points, and an energy cutoff of $80 \mathrm{eV}$ for the response function in the case of both $\mathrm{M} @ \mathrm{Si}_{12}$ and $\mathrm{M} @ \mathrm{Si}_{16}$ clusters. These criteria have been chosen in order to equally describe the electronic states of the systems. ${ }^{49}$

\section{RESULTS AND DISCUSSION}


3.1. Structure, Electric and Magnetic Properties. As expected for all $\mathrm{M} @ \mathrm{Si}_{12}$ clusters (see Fig. 1a), after geometry relaxation the $\mathrm{Si}-\mathrm{Si}$ and $\mathrm{M}-\mathrm{Si}$ bonds undergo specific distortions with respect to the symmetric cages, forming, thus, strongly bound covalent systems (see the values of relative distortion listed in Table 1). On average, the largest relative distortions with respect to the perfect $D_{6 \mathrm{~h}}$ symmetry were found for Ti@ $\mathrm{Si}_{12}(2.0 \%), \mathrm{Pd} @ \mathrm{Si}_{12}(3.4 \%)$, $\mathrm{Zr} @ \mathrm{Si}_{12}(7.5 \%)$, and $\mathrm{Hf} @ \mathrm{Si}_{12}(5.0 \%)$. These results agree with previous calculations, ${ }^{4,50}$ indicating that among all the $\mathrm{M} @ \mathrm{Si}_{12}$ clusters studied here, those belonging to group-4 of the periodic table ( $\mathrm{Ti}, \mathrm{Zr}, \mathrm{Hf}$ ), together with $\mathrm{Pd}$, give raise to the most distorted structures. Moreover, a rather distorted structure of $\mathrm{Ti} @ \mathrm{Si}_{12}$ was found by $\mathrm{He}$ et al. ${ }^{29}$ as its lowestenergy configuration, which is also in agreement with calculations conducted by Guo et al.. ${ }^{51}$ The clusters belonging to group-6 were moderately distorted, i.e., $\mathrm{Cr} @ \mathrm{Si}_{12}(0.9 \%)$ and Mo@Si $\mathrm{Si}_{12}(0.5 \%)$, while the clusters of group-8, i.e., Ru@ $\mathrm{Si}_{12}$ and Os $@ \mathrm{Si}_{12}$ became almost perfectly symmetric. The average optimized $\mathrm{Si}-\mathrm{Si}$ and $\mathrm{M}-\mathrm{Si}$ bond lengths for these clusters are listed in Table 1. In general, these distances are in very good agreement with those obtained from calculations in other works. ${ }^{4,52}$

Following the same optimization procedure discussed in the methodology section, we have obtained relaxed configurations of the $\mathrm{M} @ \mathrm{Si}_{16}$ clusters $(\mathrm{M}=\mathrm{Ti}, \mathrm{Zr}$, and $\mathrm{Hf})$. In these cases, the $C_{3 \mathrm{v}}$ symmetry (Frank-Kasper type structure) has been used as starting point for the optimization procedure (see Fig. 1b). After relaxation, the initial atomic positions did not undergo significant displacements, maintaining the symmetric Frank-Kasper shape. This result is in good agreement with recent theoretical calculations by Reis and Pacheco. ${ }^{39}$ They also reported the parameters characterizing the bond lengths as well as the bond angles of these three clusters (see Ref. ${ }^{39}$ ). The M@ $\mathrm{Si}{ }_{16}$ clusters have been successfully synthesized by experimental techniques such as laser ablation. ${ }^{19}$ and magnetron sputtering. ${ }^{53}$ Additionally, vibrational spectra calculations ${ }^{7}$ have confirmed the remarkable and higher stability of the $\mathrm{M} @ \mathrm{Si}_{16}$ cages than the stability of $\mathrm{M} @ \mathrm{Si}_{12}$. In contrast to the $\mathrm{M} @ \mathrm{Si}_{12}(\mathrm{M}=\mathrm{Ti}, \mathrm{Zr}$, and $\mathrm{Hf})$ clusters that, as mentioned above, possess significantly distorted equilibrium geometries, the corresponding $\mathrm{M} @ \mathrm{Si}_{16}$ for the same metals belonging to group-4 exhibit more symmetric shapes. As we will discuss later, the higher stability of $\mathrm{M} @ \mathrm{Si}_{16}$ also implies larger and more similar energy gaps (when these systems are compared among themselves) than in the case of the $\mathrm{M} @ \mathrm{Si}_{12}$ clusters (again, when the systems are compared within their own class).

Considering now the calculated electric properties, among all the M@Si 12 clusters analyzed here, only $\mathrm{Zr} @ \mathrm{Si}_{12}, \mathrm{Pd} @ \mathrm{Si}_{12}$ and $\mathrm{Hf} @ \mathrm{Si}_{12}$ exhibited appreciable dipole moments; i.e., 1.0, 1.2, and $0.8 \mathrm{D}$, as listed in Table 2. Interestingly, these clusters form the most distorted geometries in this series. As discussed by Wang and Han, ${ }^{50}$ the dipole moment of 
these clusters is generally related to symmetry breaking of the perfect geometry. In agreement with this assumption, our calculated dipole moment of $\mathrm{Zr} @ \mathrm{Si}_{12}$ (the most distorted structure) is $1.002 \mathrm{D}$, which is consistent with the corresponding values (1.021 D) calculated in Ref. ${ }^{50}$. Accordingly also, all the $\mathrm{M} @ \mathrm{Si}_{16}$ clusters (the least distorted structures) exhibit small dipole moments of order of 0.1 D. Note that the existence of a dipole moment in some of these clusters may be a useful feature to characterize specific clusters in the presence of an external electric field. To evaluate the amount of charge transfer (CT) between the transition metal atoms and silicon cages we have employed the Bader analysis (see Table 2). Indeed, CT is strongly dependent on the method employed to estimate the charge distribution, as well as the size and shape of the cluster, as it has long been discussed in the literature. ${ }^{50,54,55,56}$ However, the amount of CT is useful to understand the bonding character of the clusters. As can be seen in Table 2, the interaction between the transition metal and the $\mathrm{Si}_{\mathrm{n}}$ cage is characterized by substantial ionic admixtures due to $\mathrm{CT}$ between the two cluster subsystems (transition metal atom and silicon cage).

As a complement to the calculation of the dipole moment for these systems, we have also calculated their dipole polarizability, which is a valuable molecular electric property for understanding the linear optical response. Furthermore, the polarizability is closely related to the size, shape, and electronic structure of the clusters. ${ }^{29}$ Table 2 displays the calculated mean polarizabilities ${ }^{45,46}$ for all the systems studied here. Our results, as calculated within the finite field method at the PW91/PAW level of theory, have indicated that for M@ $\mathrm{Si}_{12}$ the mean polarizabilities preserve an approximate relationship with the atomic size of each encapsulated transition metal element. For example, Ti@Si $\mathrm{Si}_{12}, \mathrm{Cr} @ \mathrm{Si}_{12}$, and $\mathrm{Pd} @ \mathrm{Si}_{12}$ (in which each one of the metal atoms has similar Slater atomic radius of $140 \mathrm{pm}$ ) give mean polarizabilities of $396.20,373.57$, and 398.95 a.u., respectively. Obeying this relation, $\mathrm{Zr} @ \mathrm{Si}_{12}(\mathrm{Zr}$ atomic radius is of $185 \mathrm{pm})$ and $\mathrm{HfSi}_{12}$ (Hf atomic radius is of $155 \mathrm{pm}$ ) give mean polarizabilities of 418.72 and 415.23 a.u., respectively. In contrast, for the M@ $\mathrm{Si}_{16}$ clusters such a dependence of the polarizability on the size of the encapsulated metal atom is not observed; i.e., the calculated mean polarizabilities are almost constant regardless the metal atom involved (see Table 2). This analysis shows that for larger clusters, such as $\mathrm{M} @ \mathrm{Si}_{16}$, the mean polarizability depends much more on the volume of the silicon cage. A similar behavior for the polarizability was found by Sabirov and Bulgakov ${ }^{24}$ for distinct endofullerenes. For example, considering a $\mathrm{C}_{20}$ cage, the mean polarizability of the endofullerene increased with the increasing size of the encapsulated atom, whereas in the case of $\mathrm{C}_{60}$, the polarizability was essentially constant, regardless of the size of the encapsulated atom. ${ }^{24}$ 
In addition to the electric properties, we can analyze the caging effects on the magnetic moment of the transition metals by means of spin-polarized DFT calculations. Because of their ground state electron configurations, most of the transition metal atoms considered here exhibit net spin polarization when isolated. For example, the calculated magnetic moments of the $\mathrm{Ti}, \mathrm{Zr}$ and $\mathrm{Hf}$ atoms belonging to group-4 are 3.1, 2.0 and $2.0 \mu_{B}$, respectively. For both $\mathrm{Cr}$ and Mo (group-6), the magnetic moment is $6.0 \mu_{B}$; for both $\mathrm{Ru}$ and Os (group-8), the magnetic moment is $4.0 \mu_{B}$; and for Pd (group-10), the magnetic moment vanishes, as can be expected for its closed-shell electronic structure. Within the context of the caging effects introduced by the $\mathrm{Si}$ atoms, it is interesting to analyze the magnetic moments of these transition metals when encapsulated by the silicon cages (see Table 3 ). Upon formation of the $\mathrm{M} @ \mathrm{Si}_{n}$ clusters, their magnetic moments are completely quenched (except in the case of $\mathrm{Ti} @ \mathrm{Si}_{12}$ that still retains a magnetic moment of $2.0 \mu_{B}$ ). These results confirm that the $\mathrm{Si}$ cages significantly reduce or entirely cancel the intrinsic magnetic moment of the corresponding transition metal atoms. ${ }^{3,57,58}$ In the case of Ti@ $\mathrm{Si}_{12}$, our spinpolarized calculations have predicted the system is a spin triplet. As pointed out by Sen and Mitas $^{59}$ and also by Guo et al. ${ }^{60}$, the spin triplet ground state of the cluster has a total energy lower than its singlet state. However, He et al. ${ }^{29}$ have found a distorted spin singlet structure of $\mathrm{Ti} @ \mathrm{Si}_{12}$ with a total energy lower than the triplet state. In addition, we have found that the magnetic moment of the Si-encapsulated $\mathrm{Ti}$ atom is completely quenched when the size of its Si cage increases, as obtained in the case of Ti@Si16. This may be explained by a stronger hybridization between Ti $3 d$ and $\mathrm{Si} 3 p$ states in the Ti@ $\mathrm{Si}_{16}$ than in the distorted $\mathrm{Ti} @ \mathrm{Si}_{12}$ cluster.

3.2 Electronic States and Optical Absorption. Here, as a first estimate of the absorption gaps of these systems, we have calculated the difference between the KS eigenvalues of the highest occupied molecular orbital (HOMO) and the lowest unoccupied molecular orbital (LUMO) of their ground-state electronic structure. The values that we have obtained for the HOMO-LUMO gaps are listed in Table 3. These independent-particle gaps are in agreement with previous DFT calculations. ${ }^{2,56}$ In Figures 2 and 3, we display the calculated density of states (DOS) for the $\mathrm{M} @ \mathrm{Si}_{12}$ clusters. In order to understand the distorting effect on the electronic structure of these systems, we have separated the DOS of the most distorted clusters in Figure 2, while the DOS of the less distorted clusters are displayed in Figure 3. The charge densities of the frontier orbitals of the clusters are also displayed in their respective DOS.Ti@Si $\mathrm{Si}_{12}, \mathrm{Zr} @ \mathrm{Si}_{12}$, and $\mathrm{Hf} @ \mathrm{Si}_{12}$ exhibit HOMOs that are less symmetric and more distributed, from the metal to the silicon bonds, than the charge densities of other 
more symmetric $\mathrm{M} @ \mathrm{Si}_{12}$ clusters (see Figure 2). Regarding the LUMOs of $\mathrm{Ti} @ \mathrm{Si}_{12}$, $\mathrm{Zr} @ \mathrm{Si}_{12}$, and $\mathrm{Hf} @ \mathrm{Si}_{12}$, a significant delocalization over the silicon bonds is observed; only Ti@Si 12 's LUMO exhibits an additional density around the metal-center, leading to possible low-lying transitions mostly located at this site. In the case of Pd@ $\mathrm{Si}_{12}$, which is another cluster exhibiting a large distortion, the HOMO and LUMO are more similar among themselves, which allows low-laying transitions in this system. This feature may be responsible for the small HOMO-LUMO gap calculated for this cluster (Table 3).

The more symmetric clusters $\mathrm{Cr} @ \mathrm{Si}_{12}$ and $\mathrm{Mo} @ \mathrm{Si}_{12}$ exhibit HOMO charge densities that are less localized at the metal-center, whereas their LUMOs are highly localized at the metal. In the particular case of $\mathrm{Mo@Si} 12$, the electronic transitions should occur from an orbital of Si to an orbital of the metal, leading to a large energy gap value. Indeed, as listed in Table 3, this system exhibits the largest HOMO-LUMO gap of all the M@ $\mathrm{Si}_{12}$ clusters investigated here. This has also been confirmed by experimental measurements of the optical gap (1.05 eV) of the Mo@Si 12 film, made possible by the fact that $\mathrm{Mo} @ \mathrm{Si}_{12}$ is one of the clusters which has been synthesized and successfully deposited as an amorphous film onto silica substrates by Uchida and coworkers. ${ }^{61}$ In agreement with these results, also Sen and Mitas ${ }^{47}$ have calculated a large HOMO-LUMO gap for Mo@ $\mathrm{Si}_{12}$ (singlet state) by employing DFT schemes. Among all the $\mathrm{M} @ \mathrm{Si}_{12}$ systems considered in the present study, only Ru@Si12 and Os@Si $\mathrm{Si}_{12}$ exhibit HOMOs more definitely localized at the metal center and LUMOs delocalized towards the silicon bonds, indicating possible specific metal-silicon electronic transitions.

In Figure 4, the DOS and the charge densities near the Fermi level of the M@Si $\mathrm{Si}_{16}$ $(\mathrm{M}=\mathrm{Ti}, \mathrm{Zr}$, and $\mathrm{Hf}$ ) systems are displayed. As discussed above, these systems maintain their stable Frank-Kasper shape, in contrast to the corresponding M@ $\mathrm{Si}_{12}$ which undergo large structural distortions. In fact, when the number of silicon atoms around the transition metal atom increases, the energy gaps of the clusters become enlarged, as reported in Table 3. As displayed in Figure 3, the HOMO charge densities of the M@ $\mathrm{Si}_{16}$ are much more localized at the metal center, exhibiting a $d_{\mathrm{z}}^{2}$-like charge density typical for the transition metal atoms belonging to group-4. In general, the LUMOs of these systems are delocalized at the silicon atoms, allowing electronic transitions from an orbital belonging to the metal to an orbital of Si. The major difference in the electronic structure of these clusters occurs in the case of Ti@Si 16 , for which the LUMO also exhibits an appreciable charge density at the metal atom, which may give rise to $d$ - $d$ intrashell transitions. Such a behavior is also present for Ti@Si 12 which was discussed above. Because of the stability and characteristic features of the frontier orbitals of the $\mathrm{M} @ \mathrm{Si}_{16}$ clusters, their HOMO-LUMO gaps are more similar 
among themselves, with energies at the $2.35-2.58 \mathrm{eV}$ range. These values are in good agreement with the previous calculations by Reis and Pacheco. ${ }^{39}$

From the KS electronic states and within the RPA method, the optical absorption spectra of the $\mathrm{M} @ \mathrm{Si}_{\mathrm{n}}$ clusters were simulated in the 0-10 eV range (which includes the UVvisible region), as displayed in Figures 5-7. The calculated spectra for the less symmetric $\mathrm{M} @ \mathrm{Si}_{12}$ clusters (i.e., those including Ti, Zr, Hf, and Pd) are displayed in Figure 5. As can be seen, these spectra exhibit small absorptions at energies between 0.5 and $2 \mathrm{eV}$, while relatively intense absorptions appear between 2 and $4 \mathrm{eV}$. When compared to some available data in other works ${ }^{29}$, our results for $\mathrm{Ti} @ \mathrm{Si}_{12}$ are in a reasonable agreement, although we also determine here a far absorption region at higher energies (between 4 and $8 \mathrm{eV}$ ). Similarly, we have found intense absorption regions between 4 and $8 \mathrm{eV}$ for $\mathrm{Zr} @ \mathrm{Si}_{12}$, Hf@Si12, and Pd@Si12. Figure 6 displays the calculated spectra for the more symmetric $\mathrm{M} @ \mathrm{Si}_{12}$ clusters (i.e., those encapsulating $\mathrm{Cr}, \mathrm{Mo}, \mathrm{Ru}$, and Os). The spectra of these systems exhibit absorptions starting from approximately at $2 \mathrm{eV}$, featuring reduced absorptions at 4 $\mathrm{eV}$, while exhibiting more intense absorption between 4 and $8 \mathrm{eV}$. Finally, considering the absorption spectra of the $\mathrm{M} @ \mathrm{Si}_{16}$ clusters (Figure 7), we stress that their absorption profiles are typically more concentrated at the energies between 2 and $6 \mathrm{eV}$. As discussed above, this is a result of the band gap enlargement in the larger $\mathrm{M} @ \mathrm{Si}_{16}$ clusters.

Despite the qualitative interpretation of the probability densities of the frontier KS orbitals and the features of the absorption spectra calculated within the RPA for M@ $\mathrm{Si}_{n}$ clusters, it is well known that the independent-particle energy gap is only a first estimate of the excitation energies. Of course, it is difficult to address the effect of electronic excitations in the optical gaps of such complex systems by employing effective single particle DFT orbitals; especially because most correlation density functionals are designed specifically for the electronic ground state. Following this line of arguments, below we report more accurate estimates for the absorption gaps of the $\mathrm{M} @ \mathrm{Si}_{n}$ clusters calculated by correcting the KS energies through the $G W$ approximation.

3.3. Quasiparticle Band Gaps within $G_{\mathbf{O}}$. As a more accurate method to calculate the absorption gaps of these clusters, we have adopted the $G W$ approximation. To describe the optical gaps of the $\mathrm{M} @ \mathrm{Si}_{n}$ cages, we have considered the frequency-dependent scheme based on the PAW basis sets up to the $G W_{0}$ level of approximation. An advantage of this method is that it allows treating $d$ and $f$ orbitals with a relatively modest computational effort. The corrected HOMO-LUMO gaps within the GWA are reported in Table 3 for all the $\mathrm{M} @ \mathrm{Si}_{n}$ clusters. In Figure 8, we display the DFT and $G W_{0}$ gaps as a function of the 
atomic number of the encapsulated transition element. It is noteworthy that the $G W_{0}$ calculations widen the energy gap independently of the spatial distortion of the cluster and the atomic number of the metal atom in the cluster center. Actually, the GWA provides systematically larger values for the HOMO-LUMO gaps of all these systems.

In the case of $\mathrm{Ti} @ \mathrm{Si}_{12}, \mathrm{Zr} @ \mathrm{Si}_{12}$, and $\mathrm{Hf} @ \mathrm{Si}_{12}$, the $G W_{0}$ gaps are increased by 3.05, 2.78, and $3.15 \mathrm{eV}$, respectively, when compared to the corresponding DFT/GGA values. For the next group, $\mathrm{Cr} @ \mathrm{Si}_{12}$ and $\mathrm{Mo} @ \mathrm{Si}_{12}$, the increase is of 3.11 and $3.46 \mathrm{eV}$, respectively, whereas for $\mathrm{Ru} @ \mathrm{Si}_{12}$ and $\mathrm{Os} @ \mathrm{Si}_{12}$ the increase is of 3.23 and $2.84 \mathrm{eV}$, respectively. For $\mathrm{Pd} @ \mathrm{Si}_{12}$ this increase is of $2.32 \mathrm{eV}$, while its HOMO-LUMO gap is strongly underestimated via DFT calculations (0.13 eV with GGA, as discussed in the previous section). Among all $\mathrm{M} @ \mathrm{Si}_{12}$ clusters considered here, after corrections at the GWA level, Pd@Si 12 underwent the largest relative gap variation with respect to its DFT value. In turn, the smallest relative gap variation due to the inclusion of many-body effects was observed for Ru@ $\mathrm{Si}_{12}$. This increase is clearer in Figure 8, where we plot the energy gap as a function of the atomic number of the transition-metal center in each $\mathrm{M} @ \mathrm{Si}_{12}$ cluster.

At this point, it is also instructive to consider the size and many-body effects on the energy gap increase for the $\mathrm{M} @ \mathrm{Si}_{16}$ systems. In general, the GWA corrections lead to increased HOMO-LUMO gaps of these systems. As listed in Table 3, the increment in the GWA gaps of the M@ $\mathrm{Si}_{16}$ clusters is of $2.76-3.32 \mathrm{eV}$ from the corresponding GGA values. Notice that such increment range was similar to the calculated for the corresponding $\mathrm{M} @ \mathrm{Si}_{12}$ systems. However, it is also possible here to notice the size effect in the energy gaps of these systems. For example, whereas the GGA gaps of $\mathrm{Ti} @ \mathrm{Si}_{12}, \mathrm{Zr} @ \mathrm{Si}_{12}$, and Hf@ $\mathrm{Si}_{12}$ were respectively $0.74,0.78$, and $0.82 \mathrm{eV}$, they increase by an amount of approximately $1.5 \mathrm{eV}$ (see Table 3 ) for the corresponding M@Si16, i.e., Ti@Si16, Zr@Si16, and Hf@Si 16 . This already implies a formation of semiconducting clusters with larger gaps. If the $G W_{0}$ corrections to the DFT gaps of $\mathrm{M} @ \mathrm{Si}_{16}$ are taken into account, their energy gaps increase further by $2.76,3.32$, and $3.15 \mathrm{eV}$, respectively, thus becoming 4.99, 5.64, and 5.48 $\mathrm{eV}$, respectively. Only in the case of $\mathrm{Hf} @ \mathrm{Si}_{16}$, we have found an energy gap increment due to the $G W_{0}$ correction, which is of the same magnitude as that calculated for $\mathrm{Hf} @ \mathrm{Si}_{12}$. However, for $\mathrm{Zr} @ \mathrm{Si}_{16}$ there is a relative increase in the gap variation (due to the $G W_{0}$ correction) of $20 \%$, compared to $\mathrm{Zr} @ \mathrm{Si}_{12}$. On the contrary, for $\mathrm{Ti} @ \mathrm{Si}_{16}$ there is a reduction of $15 \%$ in the relative increase of the gap variation (due to the $G W_{0}$ correction), as compared to $\mathrm{Ti} @ \mathrm{Si}_{12}$. This effect is, however, expected due to mainly distinct QP effects in each group. 
The differences in the magnitude of the QP corrections for all these systems reveal subtle differences in the nature of their optical gaps. In Table 4, we provide the specific corrections to the HOMO and LUMO energy levels calculated with LDA. The corrected results for the HOMO-LUMO gaps can be better understood by analyzing explicitly the $G W$ corrections to the KS energy levels, i.e., $\Delta \varepsilon_{n}^{\mathrm{QP}}=\Delta\left\langle n\left|\Sigma-V_{x c}^{\mathrm{DFT}}\right| n\right\rangle$. In fact, the calculation of the screened Coulomb interaction for the $\mathrm{M} @ \mathrm{Si}_{n}$ clusters depends strongly on the type of the encapsulated metal. One may expect that if the spatial distribution of an occupied orbital is modified for each cluster, the region of strong screening would be also changed in the same direction. Thus, taking into account the spatial geometry of the Si cages in these clusters, the $G W$ corrections should be relatively stable with respect to changing the silicon distribution around the metal-center for each cluster. However, because of the distinct behavior of the electronic structure of each encapsulated transition metal and the specific hybridization between orbitals of $M$ and Si, such regularity is not observed in the case of the $\mathrm{M} @ \mathrm{Si}_{n}$ clusters.

This effect can be clarified if the difference between the LDA and $G W$ energies, i.e., $\Delta G W=\varepsilon_{n}^{\mathrm{QP}}-\varepsilon_{n}^{\mathrm{DFT}}$ is analyzed. In Table 4 , we list the $G W$ corrections to the HOMO, LUMO, and $\Delta G W$. As can be seen, there are appreciable changes in these quantities depending on the cluster examined. For example, whereas $\Delta G W \mathrm{HOMO}$ in $\mathrm{Ti} @ \mathrm{Si}_{12}$ and $\mathrm{Ti} @ \mathrm{Si}_{16}$ are essentially of the same magnitude $(-0.20$ and $-0.23 \mathrm{eV}$, respectively), in the case of $\mathrm{Zr} @ \mathrm{Si}_{12} \Delta G W$ HOMO reads $-0.64 \mathrm{eV}$, while in the case of $\mathrm{Zr} @ \mathrm{Si}_{16}$ this quantity is $-0.29 \mathrm{eV}$. These variations indicate that the number of encapsulating $\mathrm{Si}$ atoms may dramatically affect a specific energy level for a given metal-center in the cluster. For this reason, generally, it should not be expected a similar absorption pattern in the optical spectra of different-size clusters encapsulating the same metal. In this sense, the optical properties of the M@Sin clusters are dominated by many-body effects.

\section{CONCLUSIONS}

In the present work, we have theoretically determined reference values for the static dipole polarizabilities and optical linear response of $\mathrm{M} @ \mathrm{Si}_{12}(\mathrm{M}=\mathrm{Ti}, \mathrm{Cr}, \mathrm{Zr}, \mathrm{Mo}, \mathrm{Ru}, \mathrm{Pd}$, Hf, and Os) and $\mathrm{M} @ \mathrm{Si}_{16}(\mathrm{M}=\mathrm{Ti}, \mathrm{Zr}$, and Hf) clusters, within ab initio plane-wave DFT calculations. The calculations of the optical gaps were performed by employing the frequency-dependent $G W$ approximation as a post-DFT scheme. This level of theory allows the treatment of $d$ and $f$ orbitals of the $\mathrm{M} @ \mathrm{Si}_{n}$ clusters with a relatively low computational 


\section{ASSOCIATED CONTENT}

\section{Supporting Information}

Additional information on the optimized geometries of the clusters is provided in a separate document. This material is available free of charge via the Internet at http://pubs.acs.org.

\section{AUTHOR INFORMATION}

Corresponding authors

*E-mail: rivelino@ufba.br (R.R.); gekos@ifm.liu.se (G.K.G.).

\section{ACKNOWLEDGMENTS}


This work was supported by the Swedish Foundation for International Cooperation in Research and Higher Education (STINT) - Project YR2009-7017: Developing a flexible theoretical approach for designing inherently nanostructured and cluster-assembled materials. G.K.G. gratefully acknowledges support by the Linköping Linnaeus Initiative on Novel Functionalized Materials (VR). R.R. and F.de.B.M. acknowledge Conselho Nacional de Desenvolvimento Científico e Tecnológico ( $\mathrm{CNPq}$ ) for the partial support. M.I.A.O acknowledges the institutional program PDSE from CAPES - the Brazilian agency for higher education.

\section{REFERENCES}

(1) Pacheco, J. M.; Gueorguiev, G. K.; Martins, J. L. First-Principles Study of the Possibility of Condensed Phases of Endohedral Silicon Cage Clusters. Phys. Rev. B 2002, 66, 033401-1-3.

(2) Gueorguiev, G. K.; Pacheco, J. M. Silicon and Metal Nanotemplates: Size and Species Dependence of Structural and Electronic Properties. J. Chem. Phys. 2003, 199, 10313-10317.

(3) Reveles, J. U.; Khanna, S. N. Nearly-Free-Electron Gas in a Silicon Cage. Phys. Rev. B 2005, 72, 165413-1-6.

(4) Gueorguiev, G. K.; Pacheco, J. M.; Stafström, S.; Hultman, L. Silicon-Metal Clusters: Nano-Templates for Cluster Assembled Materials. Thin Solid Films 2006, 515, 1192-1196.

(5) Reis, C. L.; Martins, J. L.; Pacheco, J. M. Stability Analysis of a Bulk Material Built From Silicon Cage Clusters: A First-Principles Approach. Phys. Rev. B 2007, 76, 233406-1-4.

(6) Willand, A.; Gramzow, M.; Ghasemi, S. A. Structural Metastability of Endohedral Silicon Fullerenes. Phys. Rev. B 2010, 81, 201405-1-4.

(7) Reis, C. L.; Pacheco, J. M. Vibrational Spectra of Silicon Cage Clusters Doped With Ti, Zr, or Hf. Phys. Rev. B 2010, 82, 155440-1-6.

(8) Beck, S. M. Studies of Silicon Cluster-metal Atom Compound Formation in a Supersonic Molecular Beam. J. Phys. Chem. 1987, 87, 4233-4234.

(9) Jena, P. K.; Khanna, S. N.; Rao, B. K. Stability and Electronic Structure of Cluster Assembled Materials. Mat. Sci. Forum 1996, 232, 1-26.

(10) Haertelt, M.; Lyon, J. T.; Claes, P.; de Haeck, J.; Lievens, P.; Fielicke, A. GasPhase Structures of Neutral Silicon Clusters. J. Chem. Phys. 2012, 136, 064301-1-6.

(11) Cui, Y.; Lieber, C. M. Functional Nanoscale Electronic Devices Assembled Using Silicon Nanowire Building Blocks. Science 2001, 291, 851-853.

(12) Kumar, V. Alchemy at the Nanoscale: Magic Heteroatom Clusters and Assemblies. Comp. Mater. Sci. 2006, 36, 1-11.

(13) Hiura, H.; Miyazaki, T.; Kanayama, T. Formation of Metal-Encapsulating Si Cage Clusters. Phys. Rev. Lett. 2001, 86, 1733-1736.

(14) Uchida, N.; Miyazaki, T.; Kanayama, T. Stabilization Mechanism of $\mathrm{Si}_{12}$ Cage Clusters by Encapsulation of a Transition-Metal Atom: A Density-Functional Theory Study. Phys. Rev. B 2006, 74, 205427-1-9.

(15) Uchida, N.; Bolotov, L.; Miyazaki, T.; Kanayama, T. Stability of TaEncapsulating Si Clusters on Si(111)-(7 × 7) Surfaces. J. Phys. D 2003, 36, L43-L46. 
(16) Gueorguiev, G. K.; Stafström, S.; Hultman, L. Nano-Wire Formation by SelfAssembly of Silicon-metal Cage-Like Molecules. Chem. Phys. Lett. 2008, 458, 170-174.

(17) Ohara, M.; Koyasu, K.; Nakajima, A.; Kaya, K. Geometric and Electronic Structures of Metal (M)-Doped Silicon Clusters $(M=\mathrm{Ti}$, Hf, Mo and W). Chem. Phys. Lett. 2003, 371, 490-497.

(18) Koyasu, K.; Akutsu, M.; Mitsui, M.; Nakajima, A. Selective Formation of $M \mathrm{Si}_{16}$ $(M=\mathrm{Sc}, \mathrm{Ti}$, and V). J. Am. Chem. Soc. 2005, 127, 4998-4999.

(19) Koyasu, K.; Atobe, J.; Akutsu, M.; Mitsui, M.; Nakajima, A. Electronic and Geometric Stabilities of Clusters with Transition Metal Encapsulated by Silicon J. Phys. Chem. A 2007, 111, 42-49.

(20) Furuse, S.; Koyasu, K.; Atobe, J.; Nakajima, A. Experimental and Theoretical Characterization of $\mathrm{MSi}_{16}{ }^{-}, \mathrm{MGe}_{16}{ }^{-}, \mathrm{MSn}_{16}{ }^{-}$, and $\mathrm{MPb}_{16}{ }^{-}(M=\mathrm{Ti}, \mathrm{Zr}$, and Hf): The Role of Cage Aromaticity. J. Chem. Phys. 2008, 129, 064311-1-6.

(21) Kumar, V.; Majumder, C.; Kawazoe. $M @ \mathrm{Si}_{16}, M=\mathrm{Ti}, \mathrm{Zr}, \mathrm{Hf}: \pi$ Conjugation, Ionization Potentials and Electron Affinites. J. Chem. Phys. Lett. 2002, 363, 319-322.

(22) Iwasa, T; Nakajima, A. Geometric, Electronic, and Optical Properties of a Superatomic Heterodimer and Trimer: Sc@Si ${ }_{16}-\mathrm{VSi}{ }_{16}$ and $\mathrm{Sc} @ \mathrm{Si}_{16}-\mathrm{TiSi}_{16}-\mathrm{VSi} \mathrm{Si}_{16} . J$. Phys. Chem. C 2012, 116, 14071-14077.

(23) Kumar, V.; Kawazoe, Y. Metal-Encapsulated Fullerenelike and Cubic Caged Clusters of Silicon. Phys. Rev. Lett. 2001, 87, 045503-1-4.

(24) Sabirov, D. Sh.; Bulgakov, R. G. Polarizability Exaltation of Endofullerenes $\mathrm{X} @ \mathrm{C}_{n}(n=20,24,28,36,50$, and 60; X Is a Noble Gas Atom). JETP Lett. 2010, 92, 662665.

(25) An, Y.-P.; Yang, C.-L.; Wang, M.-S.; Ma, X.-G.; Wang, D.-H. Geometrical and Electronic Properties of the Clusters of $\mathrm{C}_{20}$ Cage Doped with Alkali Metal Atoms. J. Clust. Sci. 2011, 22, 31-39.

(26) An, Y.-P.; Yang, C.-L.; Wang, M.-S.; Ma, X.-G.; Wang, D.-H. First-Principles Study of Transport Properties of Endohedral Li@ $\mathrm{C}_{20}$ Metallofullerene. Current Appl. Phys. 2010, 10, 260-265.

(27) Wu, J.; Sun, Z.; Li, X.; Ma, B.; Tian, M.; Li, S. Theoretical Study on the Smallest Endohedral Metallofullerenes: TM@ $\mathrm{C}_{20}(T M=\mathrm{Ce}$ and $\mathrm{Gd})$. Int. J. Quantum Chem. 2011, 111, 3786-3792.

(28) Manna, D.; Ghanty, T. K. Theoretical Prediction of Icosahedral U@ $\mathrm{C}_{20}$ and Analogous Systems with High HOMO-LUMO Gap. J. Phys. Chem. C 2012, 116, 1671616725.

(29) He, J.; Wu, K.; Sa, R.; Wei, Y. (Hyper)polarizabilities and Optical Absorption Spectra of $M \mathrm{Si}_{12}$ Clusters $(M=\mathrm{Sc}-\mathrm{Zn})$ : A Theoretical Study. Chem. Phys. Lett. 2010, 490, 132-137.

(30) Williamson, A. J.; Grossman, J. C.; Hood, R. Q.; Puzder, A.; Galli, G. Quantum Monte Carlo Calculations of Nanostructure Optical Gaps: Application to Silicon Quantum Dots. Phys. Rev. Lett. 2002, 89, 196803-1-4.

(31) Delerue, C.; Lannoo, M.; Allan, G. Excitonic and Quasiparticle Gaps in Si Nanocrystals. Phys. Rev. Lett. 2000, 84, 2457-2460.

(32) Reining, L.; Onida, G.; Albrecht, S. First-Principles Approach to the Calculation of Electronic Spectra in Clusters. Comp. Mater. Sci. 1998, 10, 444-447.

(33) Rohlfing, M.; Louie, S. G. Excitonic Effects and the Optical Absorption Spectrum of Hydrogenated Si Clusters. Phys. Rev. Lett. 1998, 80, 3320-3323.

(34) Jones, R. O.; Gunnarsson, O. The Density Functional Formalism, Its Applications and Prospects. Rev. Mod. Phys. 1989, 61, 689-746.

(35) Perdew, J. P.; Zunger, A. Self-Interaction Correction to Density-Functional Approximations for Many-Electron Systems. Phys. Rev. B 1981, 23, 5048-5079. 
(36) Kresse, G.; Joubert, D. From Ultrasoft Pseudopotentials to the Projector Augmented-Wave Method. Phys. Rev. B 1999, 59, 1758-1775.

(37) Kresse, G.; Hafner, J. Ab initio Molecular Dynamics for Liquid Metals. Phys. Rev. B 1993, 47, 558-561.

(38) Kresse, G.; Furthmüller, J. Efficient Iterative Schemes for ab initio TotalEnergy Calculations Using a Plane-Wave Basis Set. Phys. Rev. B 1996, 54, 11169-11186.

(39) Reis, C. L.; Pacheco, J. M. Bulk Materials Made of Silicon Cage Clusters Doped with Ti, Zr, or Hf. J. Phys. Condens. Mat. 2010, 22, 035501-1-7.

(40) Perdew, J. P.; Wang, Y. Accurate and Simple Analytic Representation of the Electron-Gas Correlation Energy. Phys. Rev. B 1992, 45, 13244-13249.

(41) Blochl, P. E. Projector Augmented-Wave Method. Phys. Rev. B 1994, 50, 17953-17979.

(42) Shishkin, M.; Kresse, G. Implementation and Performance of the FrequencyDependent $G W$ Method Within the PAW Framework. Phys. Rev. B 2006, 74, 035101-1-13.

(43) Broyer, M.; Antoine, R.; Benichou, E.; Compagnon, I.; Dugourd, P.; Rayane, D. Structure of Nano-Objects Through Polarizability and Dipole Measurements. C. R. Physique 2002, 3, 301-317.

(44) Henkelman, G.; Arnaldsson, A.; Jónsson, H. A Fast and Robust Algorithm for Bader Decomposition of Charge Density. Comput. Mat. Sci. 2006, 36, 354-360.

(45) Brandão, I.; Rivelino, R. Fonseca, Castro, M. A. An ab initio Study of Electric Properties of Linear $(\mathrm{HCN})_{N}$ and $(\mathrm{HNC})_{N}$ Aggregates in Gas Phase. Chem. Phys. Lett. 2013, 580, 9-13.

(46) Rivelino, R.; Malaspina, T.; Fileti, E. E. Structure, Stability, Depolarized Light Scattering, and Vibrational Spectra of Fullerenols From All-Electron Density-FunctionalTheory Calculations. Phys. Rev. A 2009, 79, 013201-1-10.

(47) Shishkin, M.; Kresse, G. Self-Consistent $G W$ Calculations for Semiconductors and Insulators. Phys. Rev. B 2007, 75, 235102-1-9.

(48) Hybertsen, M. S.; Louie, S. G. Electron Correlation in Semiconductors and Insulators: Band Gaps and Quasiparticle Energies. Phys. Rev. B 1986, 34, 5390-5413.

(49) Franchini, C.; Sanna, A.; Marsman, M.; Kresse, G. Structural, Vibrational, and Quasiparticle Properties of the Peierls Semiconductor $\mathrm{BaBiO}_{3}$ : A Hybrid Functional and Self-Consistent GW+Vertex-Corrections Study. Phys. Rev. B 2010, 81, 085213-1-7.

(50) Wang, J.; Han, J. G. Geometries, Stabilities, and Electronic Properties of Different-Sized $\mathrm{ZrSi}_{\mathrm{n}}(n=1-16)$ Clusters: A Density-Functional Investigation. J. Chem. Phys. 2005, 123, 64306-1-16.

(51) Guo, L.; Liu, X.; Zhao, G. F.; Luo, Y. H. Computational Investigation of $\mathrm{TiSi}_{\mathrm{n}}$ $(\mathrm{n}=2-15)$ Clusters by the Density-Functional Theory. J. Chem. Phys. 2007, 126, 234704-17.

(52) Miyazaki, T.; Kanayama, T. First-Principles Theory for Si-Based, Atomically Thin Layered Semiconductor Crystal. Appl. Phys. Lett. 2007, 91, 082107-1-3.

(53) Lau, J. T.; Hirsch, K.; Klar, Ph.; Langenberg, A.; Lofink, F.; Richter, R.; Rittmann, J.; Vogel, M.; Zamudio-Bayer, V.; Möller, T.; Issendorff, B. v. X-Ray Spectroscopy Reveals High Symmetry and Electronic Shell Structure of Transition-MetalDoped Silicon Clusters. Phys. Rev. A 2009, 79, 053201-1-5.

(54) Guo, P.; Ren, Z.-Y.; Wang, F.; Bian, J.; Han, J.-G.; Wang, G.-H. Structural and Electronic Properties of $\mathrm{TaSi}_{n}(n=1-13)$ Clusters: A Relativistic Density Functional Investigation, J. Chem. Phys. 2004, 121, 12265-12275.

(55) Han, J.-G.; Ren, Z.-T.; Lu, B.-Z. Geometries and Stabilities of Re-Doped $\mathrm{Si}_{\mathrm{n}}(n$ $=1-12$ ) Clusters: A Density Functional Investigation. J. Phys. Chem. A 2004, 108, 51005110 .

(56) Lu, J.; Nagase, S. Structural and Electronic Properties of Metal-Encapsulated Silicon Clusters in a Large Size Range. Phys. Rev. Lett. 2003, 90, 115506-1-4. 
(57) Gambardella, P.; Dhesi, S. S.; Gardonio, C.; Grazioli, C.; Ohresser, P.; Carbone, C. Localized Magnetic States of Fe, Co, and Ni Impurities on Alkali Metal Films. Phys. Rev. Lett. 2002, 88, 047202-1-4.

(58) Khanna, S. N.; Rao, B. K.; Jena, P. Magic Numbers in Metallo-Inorganic Clusters: Chromium Encapsulated in Silicon Cages. Phys. Rev. Lett. 2002, 89, 016803-1-4.

(59) Sen, P.; Mitas, L. Electronic Structure and Ground States of Transition Metals Encapsulated in a $\mathrm{Si}_{12}$ Hexagonal Prism Cage. Phys. Rev. B 2003, 68, 155404-1-4.

(60) Guo, L.; Zhao, G.; Gu, Y.; Liu, X.; Zeng, Z. Density-Functional Investigation of Metal-Silicon Cage Clusters $\mathrm{MSi}_{n}(M=\mathrm{Sc}, \mathrm{Ti}, \mathrm{V}, \mathrm{Cr}, \mathrm{Mn}, \mathrm{Fe}, \mathrm{Co}, \mathrm{Ni}, \mathrm{Cu}, \mathrm{Zn} ; n=8-16)$. Phys. Rev. B 2008, 77, 195417-1-8.

(61) Uchida, N.; Kintou, H.; Matsushita, Y.; Tada, T.; Kanayama, T. Synthesis of New Amorphous Semiconductors Assembled from Transition-Metal-Encapsulating Si Clusters. Appl. Phys. Express 2008, 1, 121502-1-3. 


\section{Tables}

Table 1. Average Bond Lengths (in $\AA$ ) and Relative Distortion (in \%) Calculated for the $\mathrm{M} @ \mathrm{Si}_{12}$ Clusters.

\begin{tabular}{lcccc}
\hline System & $\mathrm{Si}^{-\mathrm{Si}^{a}}$ & $\mathrm{Si}^{a} \mathrm{Si}^{b}$ & $\mathrm{M}-\mathrm{Si}$ & Distortion $^{c}$ \\
\hline $\mathrm{Ti} @ \mathrm{Si}_{12}$ & 2.40 & 2.41 & 2.68 & 2.0 \\
$\mathrm{Cr} @ \mathrm{Si}_{12}$ & 2.33 & 2.37 & 2.61 & 0.9 \\
$\mathrm{Zr} @ \mathrm{Si}_{12}$ & 2.74 & 2.40 & 2.86 & 7.5 \\
$\mathrm{Mo} @ \mathrm{Si}_{12}$ & 2.37 & 2.40 & 2.66 & 0.5 \\
$\mathrm{Ru} @ \mathrm{Si}_{12}$ & 2.35 & 2.38 & 2.63 & 0.02 \\
$\mathrm{Pd} @ \mathrm{Si}_{12}$ & 2.42 & 2.36 & 2.62 & 3.4 \\
$\mathrm{Hf} @ \mathrm{Si}_{12}$ & 2.58 & 2.41 & 2.78 & 5.0 \\
$\mathrm{Os} @ \mathrm{Si}_{12}$ & 2.35 & 2.38 & 2.63 & 0.02
\end{tabular}

${ }^{a} \mathrm{Si}-\mathrm{Si}$ bond lengths within the hexagonal planes; ${ }^{b}$ Inter-hexagonal $\mathrm{Si}-\mathrm{Si}$ bond lengths. ${ }^{c}$ Average relative distortion with respect to the perfect $D_{6 \mathrm{~h}}$ symmetry.

Table 2. Dipole moments (in D), Mean Polarizability (in a.u.), and Charge Transfer (in $|e|$ ) Calculated for the M@ $\mathrm{Si}_{n}$ Clusters.

\begin{tabular}{lccc}
\hline System & Dipole moment & Mean polarizability & Charge transfer $^{a}$ \\
\hline $\mathrm{Ti} @ \mathrm{Si}_{12}$ & 0.000 & 396.20 & -1.203 \\
$\mathrm{Cr} @ \mathrm{Si}_{12}$ & 0.000 & 373.57 & -0.487 \\
$\mathrm{Zr} @ \mathrm{Si}_{12}$ & 1.002 & 418.72 & -1.207 \\
$\mathrm{Mo} @ \mathrm{Si}_{12}$ & 0.002 & 381.79 & -0.343 \\
$\mathrm{Ru} @ \mathrm{Si}_{12}$ & 0.005 & 376.38 & 0.451 \\
$\mathrm{Pd} @ \mathrm{Si}_{12}$ & 1.199 & 398.95 & 0.533 \\
$\mathrm{Hf} @ \mathrm{Si}_{12}$ & 0.844 & 415.23 & -1.241 \\
$\mathrm{Os} @ \mathrm{Si}_{12}$ & 0.000 & 375.58 & 0.789 \\
$\mathrm{Ti} @ \mathrm{Si}_{16}$ & 0.097 & 458.51 & -1.141 \\
$\mathrm{Zr} @ \mathrm{Si}_{16}$ & 0.149 & 455.47 & -1.103 \\
$\mathrm{Hf} @ \mathrm{Si}_{16}$ & 0.132 & 453.32 & -1.120
\end{tabular}

${ }^{a}$ Negative signs indicate electron transfer from the transition metal to the silicon cage. 
Table 3. GGA HOMO-LUMO Gaps, QP-Corrected Gaps (in eV), and Spin Effect (in $\mu_{B}$ ) Calculated for the M@ $\mathrm{Si}_{n}$ Clusters.

\begin{tabular}{lccc}
\hline System & GGA gap & $G W_{0}$ gap & Magnetic moment \\
\hline $\mathrm{Ti} @ \mathrm{Si}_{12}$ & 0.74 & 3.79 & 2.0 \\
$\mathrm{Cr} @ \mathrm{Si}_{12}$ & 0.86 & 3.97 & 0.0 \\
$\mathrm{Zr} @ \mathrm{Si}_{12}$ & 0.78 & 3.56 & 0.0 \\
$\mathrm{Mo} @ \mathrm{Si}_{12}$ & 1.04 & 4.50 & 0.0 \\
$\mathrm{Ru} @ \mathrm{Si}_{12}$ & 1.00 & 4.23 & 0.0 \\
$\mathrm{Pd} @ \mathrm{Si}_{12}$ & 0.13 & 2.45 & 0.0 \\
$\mathrm{Hf} @ \mathrm{Si}_{12}$ & 0.82 & 3.97 & 0.0 \\
$\mathrm{Os} @ \mathrm{Si}_{12}$ & 0.92 & 3.76 & 0.0 \\
$\mathrm{Ti} @ \mathrm{Si}_{16}$ & 2.23 & 4.99 & 0.0 \\
$\mathrm{Zr} @ \mathrm{Si}_{16}$ & 2.32 & 5.64 & 0.0 \\
$\mathrm{Hf} @ \mathrm{Si}_{16}$ & 2.33 & 5.48 & 0.0 \\
\hline
\end{tabular}

Table 4. GWA Corrections to the HOMO and LUMO Energy Levels and to the LDA Electronic Gaps (in eV) for the $\mathrm{M} @ \mathrm{Si}_{n}$ Clusters.

\begin{tabular}{ccccc}
\hline System & LDA gap & $\Delta G W$ HOMO & $\Delta G W$ LUMO & $\Delta G W$ gap \\
\hline $\mathrm{Ti} @ \mathrm{Si}_{12}$ & 0.77 & -0.20 & 2.81 & 3.02 \\
$\mathrm{Cr} @ \mathrm{Si}_{12}$ & 0.79 & -0.43 & 2.75 & 3.18 \\
$\mathrm{Zr} @ \mathrm{Si}_{12}$ & 0.75 & -0.64 & 2.16 & 2.81 \\
$\mathrm{Mo} @ \mathrm{Si}_{12}$ & 0.99 & -0.55 & 2.96 & 3.51 \\
$\mathrm{Ru} @ \mathrm{Si}_{12}$ & 1.04 & -0.13 & 3.06 & 3.20 \\
$\mathrm{Pd} @ \mathrm{Si}_{12}$ & 0.12 & -0.53 & 1.80 & 2.34 \\
$\mathrm{Hf} @ \mathrm{Si}_{12}$ & 0.85 & -0.55 & 2.57 & 3.12 \\
$\mathrm{Os} @ \mathrm{Si}_{12}$ & 0.93 & -0.48 & 2.34 & 2.82 \\
$\mathrm{Ti} @ \mathrm{Si}_{16}$ & 2.36 & -0.23 & 2.70 & 2.93 \\
$\mathrm{Zr} @ \mathrm{Si}_{16}$ & 2.43 & -0.29 & 2.90 & 3.20 \\
$\mathrm{Hf} @ \mathrm{Si}_{16}$ & 2.43 & -0.21 & 2.83 & 3.05 \\
\hline
\end{tabular}




\section{Figure Captions}

Figure 1. (a) The hexagonal cage-like prism shape $\left(D_{6 \mathrm{~h}}\right)$ of the $\mathrm{M} @ \mathrm{Si}_{12}$ clusters. (b) The Frank-Kasper type structure $\left(C_{3 \mathrm{v}}\right)$ of the $\mathrm{M} @ \mathrm{Si}_{16}$ clusters.

Figure 2. Density of states (DOS) of Ti@ $\mathrm{Si}_{12}, \mathrm{Zr} @ \mathrm{Si}_{12}$, Hf@Si $\mathrm{Si}_{12}$, and $\mathrm{Pd} @ \mathrm{Si}_{12}$. Isosurfaces of the probability densities for the Kohn-Sham eigenstates near the Fermi level calculated at the point are displayed on the DOS.

Figure 3. Density of states (DOS) of Cr@Si $\mathrm{Si}_{12}, \mathrm{Mo} @ \mathrm{Si}_{12}, \mathrm{Ru} @ \mathrm{Si}_{12}$, and Os@Si $\mathrm{Si}_{12}$. Isosurfaces of the probability densities for the Kohn-Sham eigenstates near the Fermi level calculated at the point are displayed on the DOS.

Figure 4. Density of states (DOS) of Ti@Si $\mathrm{Si}_{16}, \mathrm{Zr} @ \mathrm{Si}_{16}$, and $\mathrm{Hf} @ \mathrm{Si}_{16}$. Isosurfaces of the probability densities for the Kohn-Sham eigenstates near the Fermi level calculated at the point are displayed on the DOS.

Figure 5. Absorption spectra of Ti@Si $\mathrm{Si}_{12}, \mathrm{Zr} @ \mathrm{Si}_{12}, \mathrm{Hf} @ \mathrm{Si}_{12}$, and $\mathrm{Pd} @ \mathrm{Si}_{12}$, calculated within RPA. Dynamic polarizabilities are given in arbitrary units.

Figure 6. Absorption spectra of $\mathrm{Cr} @ \mathrm{Si}_{12}, \mathrm{Mo} @ \mathrm{Si}_{12}, \mathrm{Ru} @ \mathrm{Si}_{12}$, and Os@Si12, calculated within RPA. Dynamic polarizabilities are given in arbitrary units.

Figure 7. Absorption spectra of Ti@Si $\mathrm{Si}_{16}, \mathrm{Zr} @ \mathrm{Si}_{16}$, and $\mathrm{Hf} @ \mathrm{Si}_{16}$, calculated within RPA. Dynamic polarizabilities are given in arbitrary units.

Figure 8. Energy gap variation as a function of the atomic number $(\mathrm{Z})$ of the transitionmetal center in each $\mathrm{M} @ \mathrm{Si}_{12}$ cluster. Blue circles correspond to DFT and black triangles correspond to $G W_{0}$. 
Figure 2

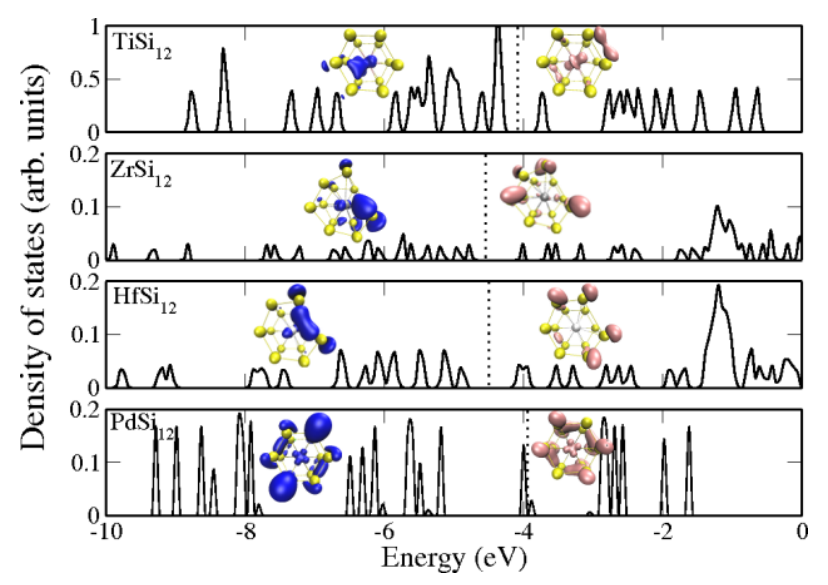

Figure 3

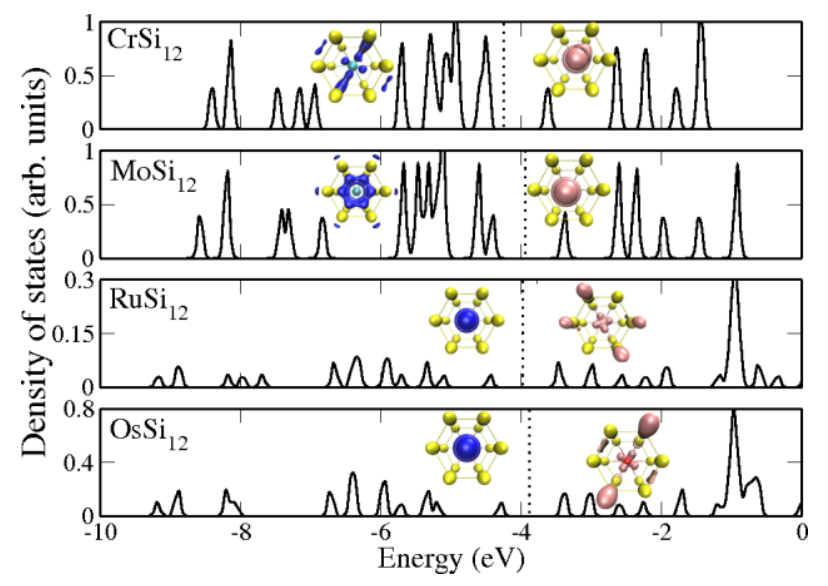


Figure 4

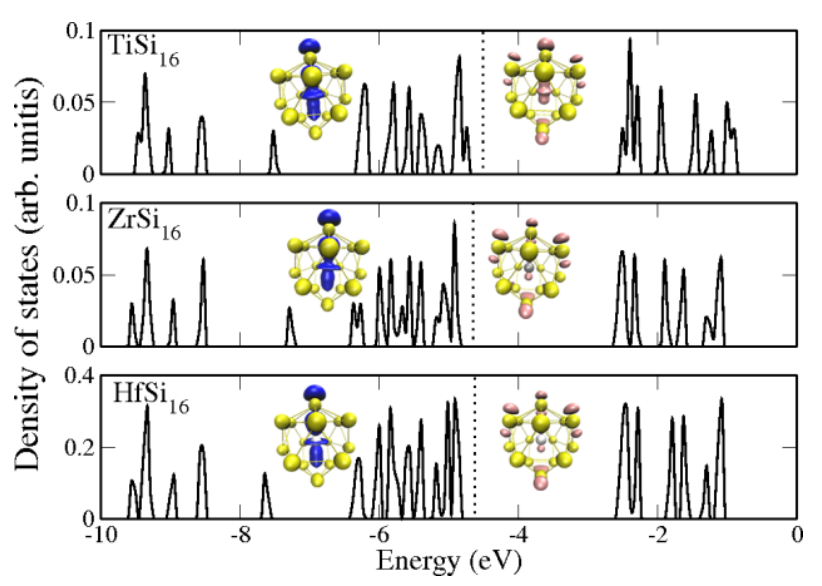

Figure 5

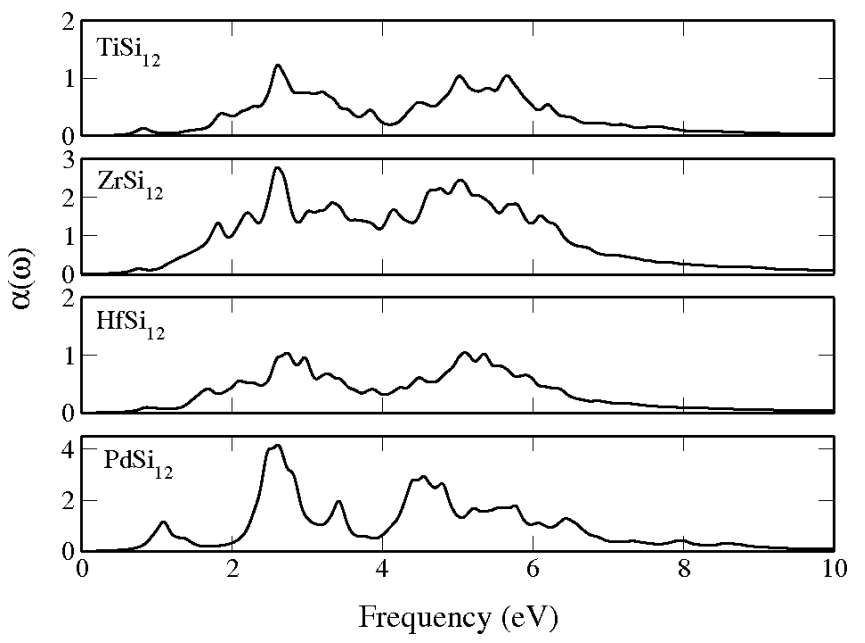

Figure 6

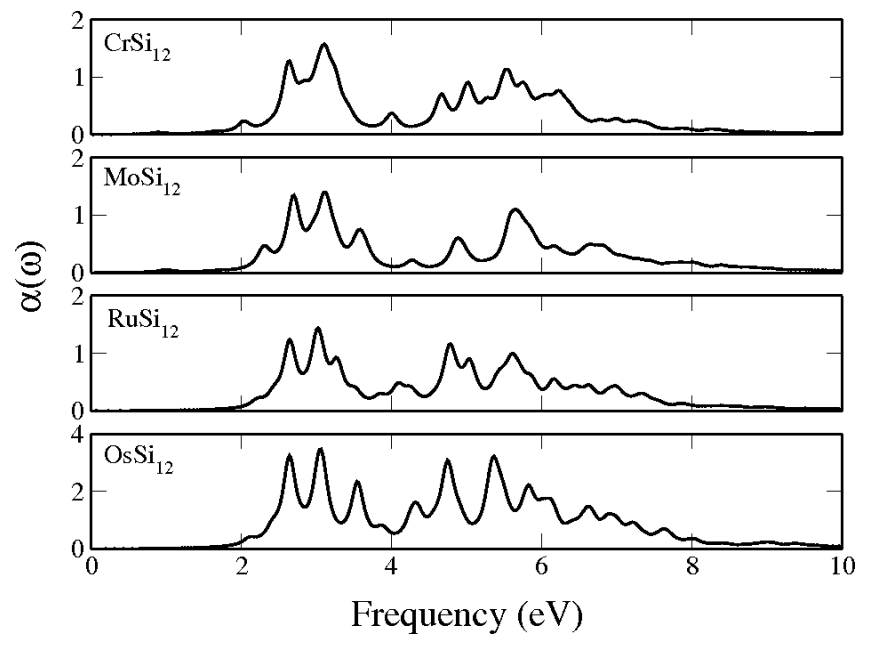


Figure 7

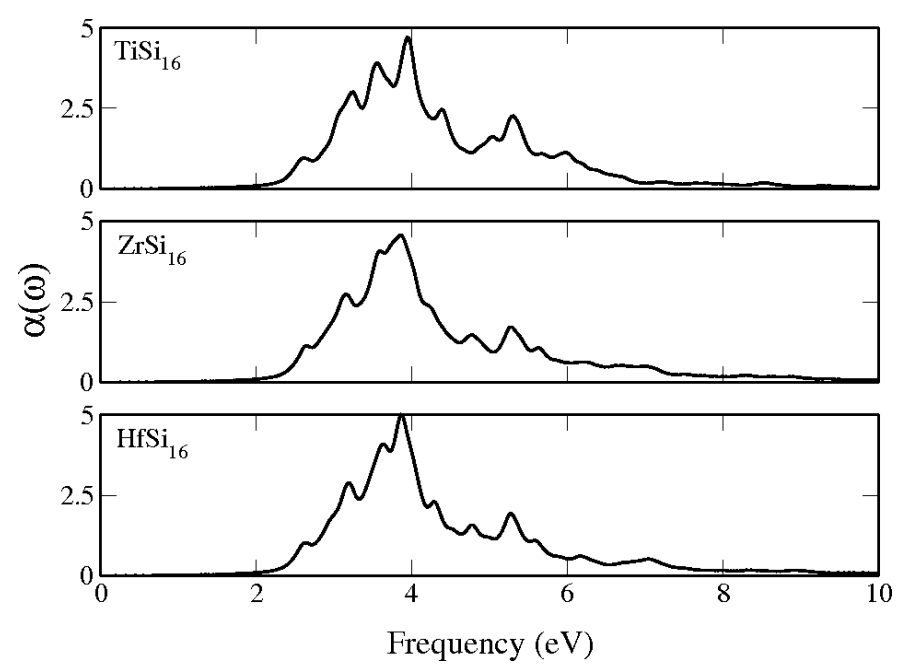

Figure 8

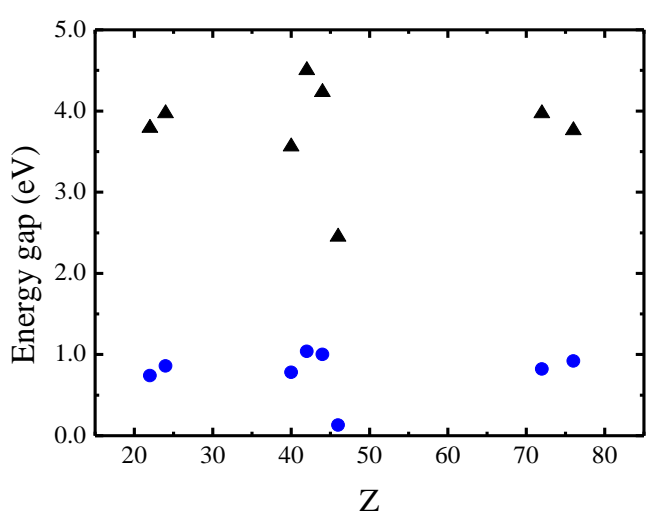


TOC

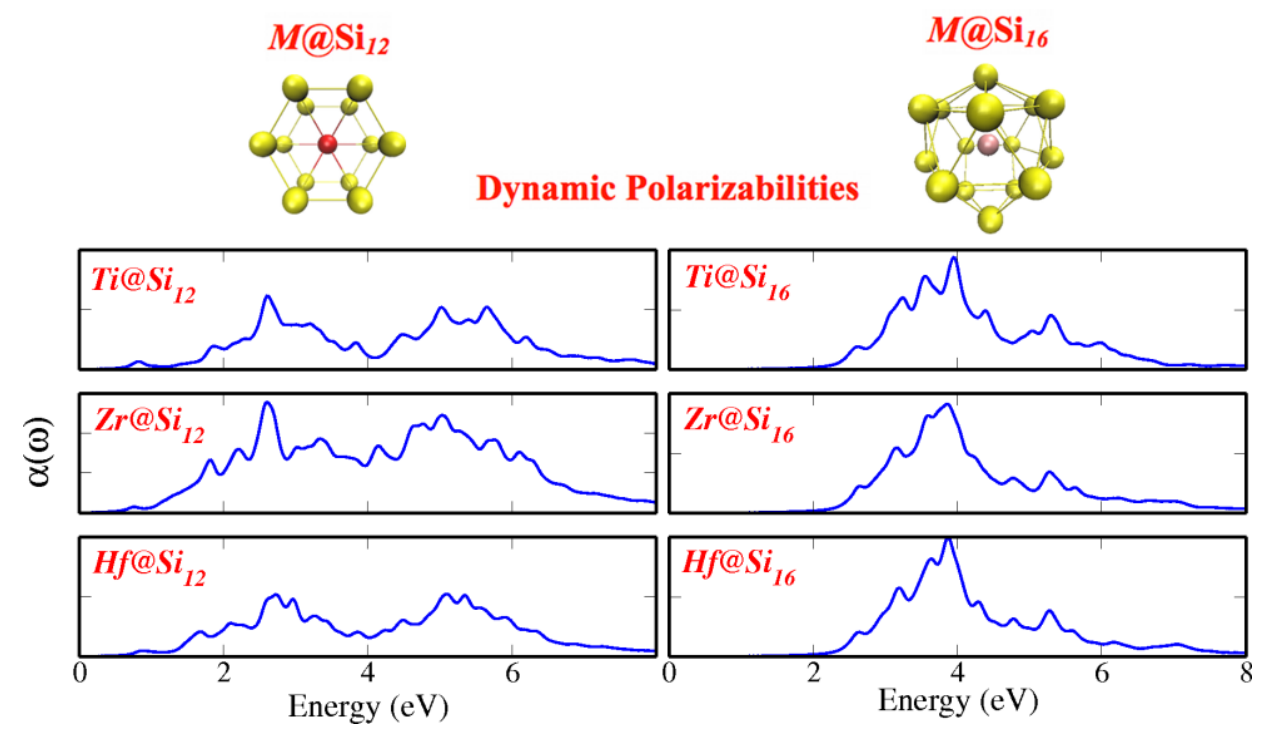

